\title{
Aus den Verhandlungen des FMH-Zentralvorstands
}

St. An seiner Sitzung vom 25. Februar 2003 behandelte der FMH-Zentralvorstand unter anderem folgende Geschäfte.

\section{Prävention}

Manual zur Beratung bei gewünschtem Schwangerschaftsabbruch

Am 1. Oktober 2002 sind die neuen Bestimmungen des Strafgesetzbuches zum straffreien Schwangerschaftsabbruch in Kraft getreten. In Zusammenarbeit zwischen FMH, Schweizerischer Gesellschaft für Gynäkologie und Geburtshilfe (SGGG), Schweizerischer Gesellschaft für Allgemeinmedizin (SGAM), Schweizerischer Gesellschaft für Innere Medizin (SGIM) und Schweizerischer Gesellschaft für Psychiatrie und Psychotherapie (SGP) wurde ein Manual erarbeitet. Dieses hat zum Ziel, die ungewollt Schwangere $\mathrm{zu}$ einer informierten, selbstbestimmten und reflektierten Entscheidung zu führen.

Der Zentralvorstand heisst das vorliegende Manual gut, stimmt dem Text für die Schweizerische Ärztezeitung [1] zu und gibt der SGGG grünes Licht zum Aufschalten des Manuals auf ihre Homepage.

\section{Aus-, Weiter- und Fortbildung}

\section{Delegierte Psychotherapie}

Bekanntlich hat das Plenum der Kommission für Weiter- und Fortbildung die Schaffung eines Fähigkeitsausweises «Delegierte Psychotherapie» abgelehnt. Mit dieser Ablehnung ist weder das Problem gelöst, noch sind die Diskussionen verstummt. Der Zentralvorstand beschliesst deshalb die Einberufung einer Konferenz zur Delegierten Psychotherapie unter Einbezug folgender Gruppierungen auf Ende März/Anfang April 2003: Schweizerische Gesellschaft für Psychiatrie und Psychotherapie, Schweizerische Gesellschaft für Kinder- und Jugendpsychiatrie und -psychotherapie, Foederatio Medicorum Psychiatricorum et Psychotherapeuticorum, Akademie für psychosomatische und psychosoziale Medizin, Interessengemeinschaft für delegierte Psychotherapie, Kollegium für Hausarztmedizin, Schweizerische Gesellschaft für Allgemeinmedizin, Schweizeri- sche Gesellschaft für Innere Medizin, Schweizerische Gesellschaft für Gynäkologie und Geburtshilfe, Schweizerische Gesellschaft für Pädiatrie und Forum Praxispädiatrie. Diese Gruppe soll die Weiterbildungsziele und -inhalte definieren, welche zur selbständigen Erbringung der ärztlichen Psychotherapie befähigen. Sie soll ausserdem definieren, welche Weiterbildungsziele und -inhalte zur Delegation der Psychotherapie an nichtärztliche Psychotherapeuten befähigen. Der Zentralvorstand erwartet einen Schlussbericht mit allfälligem Antrag zuhanden ZV bzw. Ärztekammer.

\section{Facharztprüfung im Ausland?}

Die Schweizerische Gesellschaft für Plastische, Rekonstruktive und Ästhetische Chirurgie, die Schweizerische Ophthalmologische Gesellschaft sowie die Schweizerische Gesellschaft für Klinische Pharmakologie und Toxikologie beantragen, Teile bzw. die gesamte Facharztprüfung im Ausland durchführen zu dürfen. Es handelt sich dabei um eine Ausweitung der bisher von vielen Fachgesellschaften geübten Praxis, die schriftlichen Prüfungen in der Schweiz im Rahmen europäischer Prüfungen (MC-Prüfungen für European Boards) durchzuführen. Die Durchführung der gesamten Facharztprüfung im Ausland ist die maximale Ausweitung der bisherigen Praxis. Für diese Ausweitung werden verschiedenste Gründe genannt: kleine Fachgesellschaft, die ausserstande ist, eine valable schriftliche Prüfung zu vertretbaren Kosten aufzustellen; Wunsch der jetzigen (und künftigen) Titelinhaber nach einem europäischen Diplom; das europäische Diplom ist «mehr wert» als das CH-Diplom.

Der Zentralvorstand beschliesst die Ablehnung der Anträge, solange die entsprechenden europäischen gesetzlichen Voraussetzungen und notwendigen bilateralen Verträge fehlen. Kommt dazu, dass die Weiterbildungsinhalte und -curricula innerhalb der EU zu wenig harmonisiert sind, als dass eine einheitliche Facharztprüfung geschaffen werden könnte; die entsprechenden rechtlichen Voraussetzungen fehlen. Und: Aus rechtlichen Gründen muss die Prüfungskommission der jeweiligen Fachgesellschaft die Herrschaft und damit auch die Verantwortung über die Facharztprüfung innehaben. 


\section{Organisatorisches}

Managementgrundlagencurriculum 2003 für Assistenzärztinnen und Assistenzärzte Die Bedeutung des Managements für die Tauglichkeit im Spital wie in der Praxis steht für den ZV nicht zur Diskussion. Managementkenntnisse sollten in jedes Weiterbildungsprogramm eingebaut werden. Die Halbwertszeit des Wissens verkürzt sich immer rascher; e-Lernen ermöglicht, mit diesen Veränderungen besser Schritt zu halten. Virtuelles e-Lernen stellt eine attraktive Ergänzung zum traditionellen Präsenzlernen dar und ermöglicht zudem als «Distanzlernen» eine Reduktion der Seminarpräsenz.
Das College-M bietet ein neues Managementgrundlagencurriculum an, das sowohl in der traditionellen Präsenzform als auch in der neuen Art des «Blended Learning» angeboten wird, was eine getrennte Evaluation beider Formen erlaubt.

Die FMH sollte eine Anschubfinanzierung und ein Engagement über drei bis fünf Jahre vorsehen. Dazu müssten Mitgliederbeiträge eingesetzt werden. In spätestens fünf Jahren sollte das Projekt selbsttragend sein.

Der Zentralvorstand beschliesst eine Investition auf zwei Jahre. Bei Erfolg wird das Projekt weiterfinanziert. Vorerst legt er sich auf die Variante mit Minimaltutorat fest. Nach einer Zwischenevaluation besteht die Option, allenfalls zur Maximalvariante wechseln zu können.

\section{Délibérations du Comité central de la FMH}

St. Lors de sa séance du 25 février 2003, le Comité central de la FMH (CC) s'est notamment penché sur les affaires suivantes.

\section{Prévention}

Manuel de conseils en matière d'interruption de grossesse

Les nouvelles dispositions du Code pénal concernant la dépénalisation de l'interruption de grossesse sont entrées en vigueur le $1^{\text {er }}$ octobre 2002. Un manuel a été conçu en collaboration avec la FMH, la Société suisse de gynécologie et obstétrique (SSGO), la Société suisse de médecine générale (SSMG), la Société suisse de médecine interne (SSMI) et la Société suisse de psychiatrie et psychothérapie (SSP). Cet ouvrage doit permettre à la femme enceinte souhaitant avorter de prendre une décision en toute indépendance et connaissance de cause.

Le Comité central approuve le manuel et sa publication partielle dans le Bulletin des médecins suisses [1] et donne le feu vert à la SSGO pour en livrer le contenu sur sa page internet.

\section{Formation prégraduée, postgraduée et continue}

\section{Psychothérapie déléguée}

Le plénum de la Commission pour la formation postgraduée et continue a rejeté la création d'une attestation de formation complémentaire (AFC) «psychothérapie déléguée». Cette décision ne résout pas le problème, ni ne coupe court au débat. Dès lors, le Comité central décide d'organiser une conférence sur la psychothérapie déléguée qui réunira, fin mars ou début avril 2003, les institutions suivantes: Société suisse de psychiatrie et psychothérapie, Société suisse de psychiatrie et psychothérapie d'enfants et d'adolescents, Foederatio medicorum psychiatricorum et psychotherapeuticorum, Académie de médecine psychosomatique et psychosociale, groupement d'intérêts pour la psychothérapie déléguée, Collège de médecine de premier recours, Société suisse de médecine générale, Société suisse de médecine interne, Société suisse de gynécologie et obstétrique, Société suisse de pédiatrie et Forum de pédiatrie ambulatoire. Ce groupement est habilité à définir la teneur et les objectifs de 
la formation postgraduée destinée à exercer la psychothérapie médicale de manière autonome. Il doit également définir quels sont la teneur et les objectifs de formation permettant la pratique de la psychothérapie déléguée à des psychothérapeutes non-médecins. Le Comité central attend un rapport final avec proposition éventuelle déposée à l'intention du CC ou de la Chambre médicale.

\section{Examen de spécialiste à l'étranger?}

La Société suisse de chirurgie plastique, esthétique et reconstructive, la Société suisse d'ophtalmologie et la Société suisse de pharmacologie clinique et toxicologie demandent que des parties, voire la totalité de l'examen de spécialiste, puissent être organisées à l'étranger. Il s'agit là d'une expansion répondant à une pratique déjà existante dans nombre de sociétés de discipline, visant à organiser les examens écrits suisses dans le cadre d'examens européens (examens MC des «European Boards»). La tenue complète de l'examen de spécialiste à l'étranger représente l'expansion maximale par rapport à la pratique actuelle. Plusieurs arguments plaident pour ce mode de faire, notamment le fait qu'une société de discipline de petite dimension est incapable de faire face aux frais engendrés par un examen écrit répondant aux exigences, que les porteurs de titres actuels et futurs souhaitent briguer un diplôme européen et que le diplôme européen aurait plus de «valeur» que le diplôme suisse.

Le Comité central décide de rejeter ces propositions, aussi longtemps que les conditions légales européennes et les dispositions des accords bilatéraux ne sont pas encore sous toit. A cela s'ajoute que la teneur de la formation postgraduée et ses curricula ne sont pas encore harmonisés de manière optimale au sein de l'UE afin de pouvoir créer un examen de spécialiste unifié.
Les dispositions légales nécessaires manquent, sans compter que pour des raisons d'ordre juridique, la commission d'examen de chaque société de discipline médicale a l'autorité, et donc la responsabilité en matière d'examen de spécialiste.

\section{Organisation}

\section{Curriculum en management 2003 pour les médecins-assistants}

Le CC ne conteste nullement l'importance du management dans son application en milieu hospitalier et en cabinet médical. Les connaissances dans ce domaine devraient figurer dans tout programme de formation postgraduée. La durée de vie du savoir raccourcit de plus en plus. L'apprentissage par voie électronique permet de mieux suivre les changements en cours. Cet apprentissage virtuel représente un complément attrayant à celui, traditionnel, de la présence personnelle, tout en permettant, par la distance, de réduire le nombre de séminaires à suivre sur place. College-M offre un nouveau curriculum d'apprentissage des bases du management, sous la forme aussi bien de la présence personnelle que de l'apprentissage «composite», permettant ainsi une évaluation séparée des deux. La FMH prévoit un premier financement et un engagement de trois à cinq ans pour lesquels il faudra avoir recours aux cotisations de membres. Le projet devrait être financièrement autonome dans cinq ans au plus.

Le Comité central décide un investissement sur deux ans. En cas de succès, le financement du projet sera reconduit. Le projet s'en tient pour le moment à un tutorat minimal. La première évaluation terminée, il s'agira de décider, le cas échéant, d'une option visant une variante maximale. 